Dans le BMS n 48/2012, le Swiss Medical Board a présenté deux aide-mémoire destinés à aider les patients à prendre des décisions en ce qui concerne le traitement de la rupture de ligaments croisés antérieurs et l'utilisation des tests PSA dans le dépistage précoce du cancer de la prostate [1]. Les sociétés de discipline concernées, la SSOT et la SSU, ont réagi au lancement de ces aide-mémoire avec une lettre ouverte, publiée ci-dessous. En raison de l'importance de cette question pour le corps médical, nous avons demandé à la FMH et à l'ASSM, toutes deux membres du Swiss Medical Board, de répondre à cette critique. Nous publions leur réponse à la suite du texte des sociétés de discipline.

La rédaction

1 Le Swiss Medical Board publie des aide-mémoire pour les patients. Bull Méd Suisses. 2012;93(48):1781.

\title{
Lettre ouverte
}

\section{La SGOT-SSOT et la SGU-SSU ne sont pas d'accord avec les aide-mémoire pour patients du Swiss Medical Board}

\author{
Bernhard Christen ${ }^{a}$, \\ Thomas Gasser ${ }^{b}$ \\ a Dr méd., MHA, Président de la \\ Société Suisse d'Orthopédie et \\ de Traumatologie (SGOT-SSOT) \\ b Prof. Dr, Président de la Société \\ Suisse d'Urologie (SGU-SSU)
}

\section{Correspondance:} Office SGU-SSU

15 , avenue des Planches CH-1820 Montreux

Tél. 0219632139

Fax 0219632149

office[at]cpconsulting.ch
Fin novembre, le Swiss Medical Board a publié des aide-mémoire pour patients concernant le traitement chirurgical ou conservateur de la rupture du ligament croisé antérieur et l'importance du dépistage précoce du cancer de la prostate.

Les sociétés de discipline médicale compétentes, c'est-à-dire la Société Suisse d'Orthopédie et de Traumatologie (SGOT-SSOT) et la Société Suisse d'Urologie (SGU-SSU) avaient été exclues lors de la rédaction de ces aide-mémoire. Cette manière de faire va à l'encontre de notre bonne tradition suisse qui prévoit la consultation des parties concernées.

Les deux sociétés de discipline médicale sont déçues de la qualité de ces aide-mémoire. Force est de constater qu'il y a une grande différence entre une analyse bibliographique faite par un conseil d'experts extérieurs à la discipline (comme cela a été fait par le Swiss Medical Board) et la consultation auprès du médecin.

Le résultat: des aide-mémoire peu équilibrés, incomplets, ne tenant pas compte des besoins des patients. Des aspects importants - consciemment ou inconsciemment - ont été omis. Aussi, ces aide-mémoire n'apporteront aucune aide quant aux craintes des patients, tout au contraire!

La SGOT-SSOT et la SGU-SSU refusent ces aidemémoire et conseillent à leurs membres de ne pas les utiliser. Elles demandent au corps médical de faire de même. Les organisations de patients sont également invitées de ne pas les utiliser. 